\title{
TOWARDS A MOLECULAR INTERPRETATION OF MATERIAL TRANSPORT IN POLYMERS
}

\author{
Patrick Meares
} Department of Chemistry, University of Aberdeen, Old Aberdeen AB9 $2 U E$,
Scotland

\begin{abstract}
The successful development of processes of separation by permeation through polymeric membranes requires an understanding of the simultaneous transport of two or more substances. Although the permeation of single substances has been widely studied the systematic examination of mixtures has only just begun. This paper discusses the ways in which simultaneous permeants may mutually influence one another's fluxes and thereby determine separation factors.

The separation of a binary organic liquid mixture by hyperfiltration is considered in detail. It is shown that the system may be characterized by four composition-dependent friction coefficients of which only three are probably independent. The friction coefficients may be determined from a properly designed series of interdiffusion and hyperfiltration experiments. The procedural requirements for doing this are fully explained.

The friction coefficients characterize pair-wise molecular encounters and it is probable that they will prove to be interpretable in terms of the same concepts as have been used successfully with the concentration dependent diffusion of single substances in polymers.
\end{abstract}

\section{INTRODUCTION}

The successful large scale operation of a molecular separation process based on the use of selective polymer membranes has been achieved only relatively recently. The first such process was electrodialysis for the desalination of water. It was soon followed by a second process, reverse osmosis, which had at the outset the same objective ${ }^{1}$. The subsequent development of these processes has stimulated an interest in the possibilities of separating other mixtures by membrane permeation ${ }^{2}$. Much has already been written extolling the potential advantages of such processes over conventional techniques but at the present time only very few have approached commercial viability.

The versatility of polymers as a source of thin coherent films which can have a wide range of chemical and physical properties has made them the natural choice in the search for permselective membranes ${ }^{3}$. In order to be able to separate molecules a membrane must be devoid of deliberately 
introduced pores; it must also contain a good deal of amorphous material because the permeability of the crystalline regions of polymers is too low to carry useful fluxes. Thus one is aiming to separate molecules on the basis of their size, shape and chemical properties by passage through an amorphous array of frequently randomly packed polymer molecules whose chain units are comparable in size with the molecules to be separated. It is therefore not surprising that high selectivities are not easily attained.

To represent the permeation of a single substance between two phases separates: by a membrane one may notionally write ${ }^{4}$

$$
\text { flux }=\text { force } \times \text { concentration } \times \text { mobility. }
$$

Here the force is governed by the conditions of concentration, pressure and temperature in the source and sink phases. The factor 'concentration' refers to the amount of permeate taken up by the membrane at its faces from the outer phases. 'Mobility' refers to the freedom of movement of the permeate molecules in the membrane. The first factor is therefore a process variable and not a membrane property, the second is a function of the membrane properties and of the ambient conditions, and the third is essentially a membrane property although, of course, it is a function of the temperature and, frequently, of the permeate concentration also.

When two or more permeates are present together it is usually not possible to vary the force on each independently of that on the others. The presence of each permeate may alter the solubilities and hence the concentrations of the others in the membrane even when their concentrations (or, more properly, activities or fugacities) are held constant in the outer phases. The presence of each permeate in the membrane may alter the mobilities of all the others. These complications alone would make it almost impossible to predict membrane selectivities from the permeabilities of the pure components in any but the simplest cases.

The possible coupling of fluxes presents a further problem which can arise in the permeation of mixtures. The flux of one permeate may be altered not only by the local presence of another component but also by its movement. Usually such coupling is positive, i.e. the flux of $i$ increases the flux of $k$ in the same direction and the effect is also mutual ${ }^{5}$. Coupling therefore acts to impede separation in the normal case of parallel flows.

\section{EXAMPLES OF SEPARATION PROCESSES}

It is useful to mention briefly four separation processes which have been investigated because they represent particular extreme situations. They are the separation by permeation of a mixture of simple gases, desalination of water by reverse osmosis, the separation of a mixture of organic liquids by pervaporation and the separation of organic liquids by hyperfiltration under pressure.

\section{Separation of gases}

Simple gases above their critical temperatures are relatively insoluble in polymers. They permeate films by mechanisms which differ somewhat; depending upon whether the polymer is above or below its glass tempera- 
ture ${ }^{6,7}$ but up to moderate pressures at constant temperature the permeability coefficients are independent of pressure ${ }^{8}$. This simple behaviour on the part of gases is a consequence partly of their small molecular size and partly of their low solubility and hence low concentration in the polymer. When two such gases permeate simultaneously at moderate or low pressures neither affects the permeability properties of the polymer and the flux of each gas is too low to couple appreciably with that of the other. In this case the fluxes of the components of the mixture, and hence the separation factors, can be calculated with confidence from the permeabilities of the pure gases ${ }^{9,10}$.

Even with gases the behaviour at high pressures may not be simple, particularly near the critical temperatures of the gases. The degree of separation achieved is less than predicted from the permeabilities of the individual gases. It has not yet been established what form the interaction between the permeating components takes in these circumstances ${ }^{11}$.

\section{Desalination by reverse osmosis}

In desalination by reverse osmosis ${ }^{12}$ the solubility of one component, the water in the membrane, is quite high (about 15 per cent in the grade of cellulose acetate frequently used) while the solubility of the salt in the membrane is extremely low. The salt which enters the film does so mainly as hydrated ions and owes its presence there to that of the sorbed water. The mobility of the water in the film is about 1000 times the mobility of the salt. Hence a high degree of permselectivity is to be expected and is achieved. Under operational conditions one starts with a solution in which the mole fraction of salt is usually less than 0.01 and has the objective of reducing this to 0.0001 or less. The concentration gradient of water between the feed and product solutions provides an osmotic driving force in the wrong direction. The water flow is driven by the applied pressure and can be varied at will within the designed range. The concentration gradient of the salt provides a large driving force, of the order $R T \ln (0.01 / 0.0001)$, which has the effect of leaking salt from the feed into the product. The applied pressure makes only a small addition, perhaps two per cent, to this diffusion force on the salt.

If the concentrations and mobilities of the water and salt are measured under conditions of zero flow by using radiotracers or conductance measurements it is found that the salt flux predicted under reverse osmosis conditions is usually less than that observed ${ }^{13}$. This is an indication that the flows of salt and water are coupled in the membrane.

Evidence regarding the nature of this coupling is provided by the additional observation that the flux of water under pressure is $2-3$ times greater in a cellulose acetate membrane than can be accounted for by its tracer diffusion coefficient in the membrane ${ }^{14,15}$. It has been concluded that the pressure driven flow of water has a hydrodynamic component. This means that momentum transfer between water molecules flowing in the membrane is more efficient than it would be if they followed random pathways while molecularly dispersed in the amorphous polymer. The ions from the salt will be concentrated into those regions of the membrane where water molecules are most likely to be found. The ions therefore share in this momentum transfer process and the coupling of their flow with that of the water may be pictured as a form of mutual frictional interaction at the molecular level. 


\section{Pervaporation}

In pervaporation ${ }^{2,16}$ a mixture of a pair of volatile organic liquids is placed in contact with one side of a polymer membrane, often semicrystalline or crosslinked, so that it can swell without disintegration. The downstream side of the membrane is maintained under vacuum and the permeates emerge as vapour at this low pressure side. Often the liquids in the mixture are rather similar in properties and volatility. In contrast with reverse osmosis, it is found that no substantial gain in separation is achieved by pressurising the liquid phase.

To achieve reasonable fluxes the liquids must swell the membrane and, under cperational conditions, their concentrations in the membrane, both absolute and relative, will vary continuously from the feed to the product side.

The mobilities or diffusion coefficients of substances which swell a polymer are strongly, often almost exponentially, dependent on their concentrations in the polymer. The flux of either liquid by itself can be measured and it has been found that the concentration dependence of the flux is quite well accounted for in terms of the effect of the liquid on the free volume of the polymer phase ${ }^{17-19}$.

When two swelling liquids are present together they may affect one another's ability to swell the membrane and furthermore the thermodynamics of such a three component system including an amorphous polymer is highly complex. In particular, it seems certain that because each liquid contributes to the free volume of the polymer phase each will contribute a factor to the diffusion coefficient/concentration inter-relationship of the other.

Very little is known about this mutual interference at present except for a few observations on the variation of the diffusion coefficient of a substance in a polymer with the concentration of a plasticizer. A plasticizer may be thought of as an additional component but of zero flux and hence of zero flux coupling. It has been found that a plasticizer always increases the diffusion coefficients of other substances in a polymer ${ }^{20}$.

Separation factors in pervaporation cannot yet be predicted from permeability data on the individual components. Until the free volume or some other theory of concentration dependent diffusion has been satisfactorily extended to such mixed systems ${ }^{21}$ it will not be possible to assess how far coupling between the fluxes of the components is also a factor influencing separation. Intuitively one feels this factor may be important in membranes where the molecular morphology has been manipulated by swelling and annealing procedures ${ }^{22,23}$.

\section{Hyperfiltration of organic liquids}

An alternative to pervaporation for the separation of mixtures of organic compounds by membrane permeation is hyperfiltration. In this process the feed and product streams are both liquids and pressure is applied to the feed stream as the driving force. To date this process has been studied only superficially and then mainly with cellulose acetate membranes designed for water desalination ${ }^{24}$. It appears to offer two advantages over pervaporation. The driving pressure is in the control of the operator and, because both sides of the membrane are in contact with liquid phases, distortion and stress in 
the membrane due to non-uniform swelling are likely to be less in hyperfiltration than in pervaporation.

The potentialities of this separation process should now be examined systematically with a wide range of membranes. Such membranes must be able to withstand the applied pressure while giving a useful flux and good separation factors. Previous considerations have indicated they are likely to contain much amorphous material which will swell considerably and absorb preferentially the components to be separated. It is likely that in many cases differences in uptake will contribute more to permselectivity than differences in mobility. The swollen material will be in the rubbery state so the strength of the membrane will depend upon crosslinks and very probably on the presence of crystallites of other regions which bind together many chains. Polymer blends are unlikely to be useful if they are purely physical mixtures because in long service they would develop internal gradients of polymer distribution. Crosslinked blends and copolymers are expected to find application. Block and graft copolymers are particularly promising because of the different ways in which the polymeric components may interact with one another and with the substances to be separated.

A major purpose of this paper is to indicate how such an investigation could be mounted so as to obtain not only data on separation factors but also a fundamental understanding of the molecular phenomena involved in the transport of mixtures of organic substances.

\section{MOLECULAR FRICTION AND HYPERFILTRATION}

Attention will be restricted to a binary mixture so that the basic problems can be formulated in simple terms. The feed solution in which the components 1 and 2 have mole fractions $x_{1}^{0}$ and $x_{2}^{0}$ will be absorbed by the membrane at its ingoing face. The concentrations of the components in the polymer at this face, on a mole per unit volume basis, are $c_{1}^{0}$ and $c_{2}^{0}$. In any volume element distant $x$ through the membrane from the ingoing face the concentrations are $c_{1}(x)$ and $c_{2}(x)$. Under the prevailing conditions, which are assumed to be isothermal and steady, the flux densities of the components are $\phi_{1}$ and $\phi_{2}$.

The mean net velocities of the molecules of types 1 and 2 relative to the stationary membrane in the volume element under consideration are $\phi_{1} / c_{1}$ and $\phi_{2} / c_{2}$ respectively. In these steady conditions the driving forces are $\left(\partial \mu_{1} / \partial x\right)$ and $\left(\partial \mu_{2} / \partial x\right)$, where $\mu_{i}$ is the chemical potential of $i$ and includes chemical activity and pressure $\times$ volume terms. [N.B. It is convenient to define fluxes as positive in the direction opposite from that chosen for measuring increases in potentials. This definition obviates many minus signs in the equations and will be adopted throughout this paper.]

The driving forces are exactly balanced by retarding forces. These may, to a good approximation, be represented by the laws of linear friction ${ }^{25}$. The force balance equations can then be written

$$
\left(\frac{\partial \mu_{1}}{\partial x}\right)_{T}=f_{1 \mathbf{p}}\left(\frac{\phi_{1}}{c_{1}}\right)+f_{12}\left(\frac{\phi_{1}}{c_{1}}-\frac{\phi_{2}}{c_{2}}\right)
$$




$$
\left(\frac{\partial \mu_{2}}{\partial x}\right)_{T}=f_{2 \mathrm{p}}\left(\frac{\phi_{2}}{c_{2}}\right)+f_{21}\left(\frac{\phi_{2}}{c_{2}}-\frac{\phi_{1}}{c_{1}}\right)
$$

Here the coefficients $f_{1 \mathrm{p}}$ and $f_{2 \mathrm{p}}$ give the frictional forces per mole of components 1 and 2 with the polymer in their vicinity at unit relative velocity. $f_{12}$ and $f_{21}$ describe the frictional interaction between components 1 and 2 . The balance of forces requires

$$
f_{12} / c_{2}=f_{21} / c_{1}
$$

These frictional coefficients refer to fundamental binary interactions between moving molecules. We wish to be able to determine them and to deduce from them information on the molecular processes in the membrane. Experience in the interpretation of concentration dependent diffusion in polymers ${ }^{18}$ encourages the view that $f_{1 \mathrm{p}}$ may be connected with a coefficient extrapolated to zero concentration in the polymer $f_{1 \mathrm{p}}^{0}$ which may be given by $D_{1}^{0} / R T$ where $D_{1}^{0}$ is the diffusion coefficient of 1 in the polymer extrapolated to zero concentration. Also involved in $f_{1 \mathrm{p}}$ will be the volume fraction $v_{\mathrm{p}}$ of polymer in the element where the concentrations are $c_{1}$ and $c_{2}$. Finally the fractional free volume in the element $v_{f}$ must be involved. This would be assessed as is usual in the free volume treatment of concentration dependent diffusion in rubbery polymers but would include contributions from the polymer and from both mobile components 1 and 2 .

Similar considerations would apply to $f_{2 \mathrm{p}}$ except that $D_{2}^{0}$ would replace $D_{1}^{0} \cdot v_{\mathrm{p}}$ and $v_{f}$ have the same values for component 2 as for component 1.

The coupling term $f_{12} / c_{2}$ may prove to be correlated with $\boldsymbol{R T}$ times the reciprocal of the interdiffusion coefficient of 1 and 2 in the liquid phase. A correction would have to be made for the difference between the internal pressure in the liquid and that effective in the swollen polymer. This might also be represented as a function of the local free volume.

If the components 1 and 2 are not randomly distributed in the polymer but tend for example to cluster together into easily swollen or lightly crosslinked regions then $f_{12} / c_{2}$ may be larger than expected while $f_{1 \mathrm{p}}$ and $f_{2 \mathrm{p}}$ would be smaller. These remarks indicate the ways in which frictional coefficients can be used to diagnose molecular mechanisms but further speculation is futile without data for this hyperfiltration process.

\section{THE DETERMINATION OF FRICTIONAL COEFFICIENTS}

The determination of fluxes $\phi_{1}$ and $\phi_{2}$ and of equilibrium sorbed concentrations $c_{1}^{0}$ and $c_{2}^{0}$ as functions of the liquid feed composition and pressure involves experimental effort but is not especially difficult. The determination of the frictional coefficients from these data is now, however, straightforward. The left hand sides of equations (1) and (2) are the local gradients of chemical potential whereas one can measure directly only macroscopic differences $\Delta \mu_{1}$ and $\Delta \mu_{2}$ across the membrane. An integration of equations (1) and (2) would require that the concentration profiles of the components across the membrane be known.

This difficulty may be overcome by proceeding in a different way now familiar in the non-equilibrium thermodynamic formulation of membrane 
processes $^{26}$. Over the range for which linear relations hold between fluxes and forces one may write

$$
\begin{aligned}
& \partial \mu_{1} / \partial x=r_{11} \phi_{1}+r_{12} \phi_{2} \\
& \partial \mu_{2} / \partial x=r_{21} \phi_{1}+r_{12} \phi_{2}
\end{aligned}
$$

where the $r_{i k}$ are resistance coefficients. Onsager's reciprocity rule

$$
r_{12}=r_{21}
$$

is expected to hold for the cross term but is not assumed.

By correlating equations (1) and (2) with (4) and (5) it is established from the frictional model that

$$
\begin{gathered}
r_{12}=-f_{12} / c_{2}=-f_{21} / c_{1}=r_{21} \\
r_{11}=\left(f_{12}+f_{1 \mathrm{p}}\right) / c_{1} \\
r_{22}=\left(f_{21}+f_{2 \mathrm{p}}\right) / c_{2}
\end{gathered}
$$

must hold.

A reciprocal set of linear equations between the fluxes and forces can also be written

$$
\begin{aligned}
& \phi_{1}=l_{11}\left(\partial \mu_{1} / \partial x\right)+l_{12}\left(\partial \mu_{2} / \partial x\right) \\
& \phi_{2}=l_{21}\left(\partial \mu_{1} / \partial x\right)+l_{22}\left(\partial \mu_{2} / \partial x\right)
\end{aligned}
$$

The usual relation exists between the linear coefficients of these two sets of equations i.e.

$$
r_{i k}=A_{i k} /|l|
$$

where $A_{i k}$ is the cofactor of $l_{i k}$ and $|l|$ is the determinant of the $l_{i k}$ coefficients. Thus once the $l_{i k}$ have been determined the frictional coefficients can be evaluated without difficulty.

The $l_{i i}$ coefficients can be regarded as permeabilities while the $l_{i k}$ cross coefficient accounts for the coupling between the flows of $i$ and $k$. The reciprocity rule

$$
l_{i k}=l_{k i}
$$

should apply.

The $l_{i k}$ are local coefficients and so are functions of the local composition. It will be shown in what follows how they can be determined from a properly designed experimental study of an intrinsically homogeneous membrane.

By intrinsically homogeneous is meant a membrane whose local properties are not an explicit function of the positional coordinate $x$ as they are, for example, in a membrane with an inbuilt concentration gradient of grafted side chains. In the presence of concentration gradients of mobile components the local properties are implicit functions of the positional coordinate only because they are functions of the local composition and other intensive variables, particularly the temperature and, less drastically, the pressure.

These criteria may hold for a Loeb membrane to the extent that the two-layer model ${ }^{27}$ holds because the active layer is usually regarded as 
intrinsically homogeneous and the porous layer as being a highly permeable non-selective support for the active layer. It must be doubtful however whether this type of membrane will prove useful with mixtures of liquids which swell the active layer extensively unless the porous support layer can be composed of some chemically different polymer.

\section{PARTICLE AND PRACTICAL PERMEABILITY COEFFICIENTS}

The permeation of a binary liquid mixture through an intrinsically homogeneous membrane is considered under conditions where the permeability properties are a function of local composition but not of the gradient of composition. This means that the local permeabilities are not affected by stresses arising from gradients of swelling. At this stage the dependence of permeability on applied pressure will be ignored but this is discussed later. The treatment is adapted from our theory of ionic membranes ${ }^{28}$.

In practice measurements are made on discontinuous systems in which a membrane separates two uniform solutions which can be subject to detailed observation. Fluxes are measured by observing the entry into or exit from these solutions of the components while various control devices can be used to hold the compositions of the solutions constant ${ }^{29}$. The observable driving forces are the differences between the chemical activities $a_{1}$ and $a_{2}$ of the components in the solutions and the pressure difference across the membrane $p$ [because the dependence of permeabilities on absolute pressure is being ignored we are concerned only with pressure differences and the abbreviation of writing $p$ in place of $\Delta p$ is permissible].

The dissipation function of this membrane system is

$$
T\left(\mathrm{~d} S_{i} / \mathrm{d} t\right)=A\left(\phi_{1} \Delta \mu_{1}+\phi_{2} \Delta \mu_{2}\right)
$$

where $A$ is the membrane area and $\mathrm{d} S_{i} / \mathrm{d} t$ is the rate of entropy production due to the irreversible transport processes. The associated linear flux equations which have conjugated flows and forces are

$$
\begin{aligned}
& \phi_{1}=L_{11} \Delta \mu_{1}+L_{12} \Delta \mu_{2} \\
& \phi_{2}=L_{21} \Delta \mu_{1}+L_{22} \Delta \mu_{2}
\end{aligned}
$$

Here the $L_{i k}$ are average coefficients across the membrane and depend not only on the nature of the membrane and the temperature but also on its thickness and the compositions of the solutions.

Because $\Delta \mu_{1}$ and $\Delta \mu_{2}$ are connected through the Gibbs-Duhem equation, the flux equations (15) and (16) do not suggest convenient experiments for determining the $L_{i k}$ coefficients. This problem can be overcome by carrying out a linear transformation on the fluxes and forces in such a way as to leave the dissipation function of the system unchanged. This is done by arbitrarily selecting one component, say 1 , as solute leaving component 2 as solvent. The transformed fluxes are then chosen to be the solute flux $\phi_{1}$ and the volume flux $\phi_{\mathrm{v}}$ which is defined by

$$
\phi_{\mathrm{v}}=\phi_{1} V_{1}+\phi_{2} V_{2}
$$

$V_{1}$ and $V_{2}$ are the partial molar volumes of the components. To preserve 
formal simplicity we shall ignore changes in the partial molar volumes of the components due either to volume changes on mixing or to their compressibility under the applied pressure. This approximation has negligible quantitative significance in all foreseeable circumstances in which the theory might otherwise hold. This transformation can be expressed in the form

$$
\left(\begin{array}{l}
\phi_{1} \\
\phi_{v}
\end{array}\right)=\left(\begin{array}{ll}
1 & 0 \\
V_{1} & V_{2}
\end{array}\right) \cdot\left(\begin{array}{l}
\phi_{1} \\
\phi_{2}
\end{array}\right)
$$

The forces $X_{1}$ and $X_{v}$ which conjugate with the fluxes $\phi_{1}$ and $\phi_{v}$ are obtained by using the transformed inverse square matrix from equation (18). Thus

$$
\left(\begin{array}{l}
X_{1} \\
X_{v}
\end{array}\right)=\left(\begin{array}{cc}
1 & -V_{1} / V_{2} \\
0 & 1 / V_{2}
\end{array}\right) \cdot\left(\begin{array}{c}
\Delta \mu_{1} \\
\Delta \mu_{2}
\end{array}\right)
$$

This operation gives

$$
\begin{gathered}
X_{1}=\Delta \mu_{1}-V_{1} \Delta \mu_{2} / V_{2} \\
X_{\mathrm{v}}=\Delta \mu_{2} / V_{2}
\end{gathered}
$$

To allow for the pressure difference across the membrane the chemical potentials are written in the form

$$
\Delta \mu_{i}=\left(\Delta \mu_{i}\right)_{p, T}+p V_{i}
$$

where $\left(\Delta \mu_{i}\right)_{p, T}$ is the concentratjon dependent part of $\Delta \mu_{i}$. An osmotic pressure difference $\pi$ may be defined by

$$
\pi=-\left(\Delta \mu_{2}\right)_{p, T} / V_{2}
$$

This definition is made for the sake of convenient formulation and not because the membrane is to be imagined as semipermeable. Equations (22) and (23) give

$$
\Delta \mu_{2}=V_{2}(p-\pi)
$$

while $\left(\Delta \mu_{2}\right)_{p, T}$ is obtained from

$$
\left(\Delta \mu_{2}\right)_{p, T}=R T \Delta \ln a_{2}=R T \Delta \ln \left(f_{2} x_{2}\right)
$$

where $f_{2}$ is the rational activity coefficient in the solution phases. For many pairs of organic liquids $\Delta \ln f_{2}$ will be negligible.

A parameter with the dimensions of molar concentration $\bar{C}_{1}$ is defined by

$$
\bar{C}_{1}=-\frac{1}{V_{2}}\left(\frac{\Delta \mu_{2}}{\Delta \mu_{1}}\right)_{p, T}=\frac{\pi}{\left(\Delta \mu_{1}\right)_{p, T}}=\frac{\pi}{R T \Delta \ln a_{1}}
$$

Equations (20)-(26) now permit the new forces to be expressed in the forms

$$
\begin{aligned}
& X_{1}=\pi\left(1+\bar{C}_{1} V_{1}\right) / \bar{C}_{1} \\
& X_{V}=(p-\pi)
\end{aligned}
$$


The linear flux equations when written in terms of these new variables are

$$
\begin{aligned}
& \phi_{1}=L_{\pi} \pi\left(1+\bar{C}_{1} V_{1}\right) / \bar{C}_{1}+L_{\pi \mathrm{p}}(p-\pi) \\
& \phi_{\mathrm{v}}=L_{\mathrm{p} \pi} \pi\left(1+\bar{C}_{1} V_{1}\right) / \bar{C}_{1}+L(p-\pi)
\end{aligned}
$$

In these equations the forces can be varied independently and the effects of such variations on the fluxes $\phi_{1}$ of component 1 and $\phi_{\mathrm{v}}$ of total volume can be observed and used to evaluate the $L_{\alpha \beta}$ coefficients.

\section{DIFFERENTIAL PHENOMENOLOGICAL COEFFICIENTS}

Equations (29) and (30) hold only close to equilibrium in the range where the $L_{\alpha \beta}$ coefficients are independent of the forces. It is known however that these coefficients are functions of the average concentration in the membrane and hence of the concentration difference across the membrane. Thus there will be an apparent dependence of the $L_{\alpha \beta}$ coefficients on $\pi$ because $\pi$ cannot be altered without altering the composition difference across the membrane. This type of non-linearity, which may be called global, does not imply local non-linearity, i.e. it does not mean that the local composition dependent permeabilities are dependent on the local composition gradients. In the following paragraphs it will be shown that the true values of the composition dependent coefficients, which give rise to the average $L_{\alpha \beta}$ coefficients, can be evaluated from a suitably designed set of experimental measurements. These true coefficients will be denoted by $\mathscr{L}_{\alpha \beta}$.

In order to make clear the principles of the method a simple interdiffusion experiment under zero pressure difference will be considered. In this the opposite faces of the membrane are bathed with solutions maintained at constant compositions such that there is a concentration difference of component 1 relative to component 2 across the membrane. The components will interdiffuse across the membraneand it will be assumed that once steady permeation fluxes have been established thermodynamic equilibrium exists across each membrane/solution interface. In these circumstances the fluxes are controlled entirely by transport processes occurring within the membrane.

The fluxes in this experiment can be expressed by the appropriate forms of equations (29) and (30). They are

$$
\begin{aligned}
& \left(\phi_{1} / \pi\right)_{p=0}=L_{\pi}\left(1+\bar{C}_{1} V_{1}\right) / \bar{C}_{1}-L_{\pi \mathrm{p}} \\
& \left(\phi_{\mathrm{v}} / \pi\right)_{p=0}=L_{\mathrm{p} \pi}\left(1+\bar{C}_{1} V_{1}\right) / \bar{C}_{1}-L_{\mathrm{p}}
\end{aligned}
$$

If experiments were carried out with only a very small difference between the compositions of the solution phases, say between $C_{1}$ and $\left(C_{1}+\mathrm{d} C_{1}\right)$, the values of the $L_{\alpha \beta}$ coefficients would refer unambiguously to the membrane swollen to equilibrium with liquid mixture of composition $C_{1}$. [N.B. $C_{1}$ is a volume concentration and is related to $C_{2}$ by $C_{1} V_{1}+C_{2} V_{2}=1$ while $\left.x_{1}=C_{1} /\left(C_{1}+C_{2}\right) \cdot\right]$ Coefficients related to the experimental conditions of vanishing concentration gradient can be thought of as differential coefficients of the discontinuous membrane system. They correspond with the quantities we have called $\mathscr{L}_{\alpha \beta}$.

Consider a series of flux measurements made under the condition $p=0$ 
and with the concentration of one side of the membrane held constant at $C_{1}^{0}$. The concentration on the other side $C_{1}$ is varied. The fluxes $\phi_{1}$ and $\phi_{v}$ are functions of $C_{1}$ and can be plotted against this quantity. A plot of $\phi_{1}$ may be taken as an example.

Its slope $\left(\partial \phi_{1} / \partial C_{1}\right)_{C_{1}^{o}, p=0}$ taken at some particular concentration $C_{1}$ defines a permeability $q_{1}\left(C_{1}\right)$ which is a function of $C_{1}$ but not of $C_{1}^{0}$ provided the membrane is intrinsically homogeneous. The flux which would be observed between solutions $C_{1}^{0}$ and $C_{1}^{\prime \prime}$ can be expressed in terms of this permeability by

$$
\begin{aligned}
\phi_{1}\left(C_{1}^{0} \rightarrow C_{1}^{\prime \prime}\right) & =\int_{C_{1}^{0}}^{C_{1}^{\prime \prime}} q_{1}\left(C_{1}\right) \mathrm{d} C_{1} \\
& =\int_{C_{1}^{0}}^{c_{1}^{i}} q_{1}\left(C_{1}\right) \mathrm{d} C_{1}+\int_{C_{1}^{\prime}}^{c_{1}^{\prime}} q_{1}\left(C_{1}\right) \mathrm{d} C_{1} \\
& =\phi_{1}\left(C_{1}^{0} \rightarrow C_{1}^{\prime}\right)+\phi_{1}\left(C_{1}^{\prime} \rightarrow C_{1}^{\prime \prime}\right)
\end{aligned}
$$

where $C_{1}^{\prime}$ is any other concentration.

Equation (33) demonstrates the existence of an additivity principle which has been verified experimentally for solute and volume fluxes. It permits curves of $\phi_{1}\left(C_{1}^{0} \rightarrow C_{1}\right)$ and $\phi_{v}\left(C_{1}^{0} \rightarrow C_{1}\right)$ to be built up in a series of steps from flux measurements made over a set of contiguous concentration intervals so as to cover a range up to some concentration $C_{1}{ }^{\max }$, although the linear equations might not hold in a single experiment carried out over the concentration interval $\left(C_{1}^{0} \rightarrow C_{1}^{\text {max }}\right)$.

The force $\pi$ is a function of $C_{1}^{0}$ and $C_{1}$ only, at constant temperature. Curves of $\phi_{1}$ and $\phi_{\mathrm{v}}$ versus $C_{1}$ can be converted to $\phi_{1}$ and $\phi_{\mathrm{v}}$ versus $\pi$ if the mixtures of 1 and 2 can be considered ideal or their thermodynamic properties are known. The slope of a plot of $\phi_{1}$ versus $\pi$ can be written

$$
\left[\partial \phi_{1}\left\{C_{1}^{0}, \pi\left(C_{1}^{0}, C_{1}\right)\right\} / \partial \pi\right]_{c_{1}{ }^{0}, p=0} .
$$

If in equation (33) $C_{1}^{\prime}$ is set at $C_{1}$ and $C_{1}^{\prime \prime}$ is set at $\left(C_{1}+\mathrm{d} C_{1}\right)$ it is seen that

$$
\left[\frac{\partial \phi_{1}\left\{C_{1}^{0}, \pi\left(C_{1}^{0}, C_{1}\right)\right\}}{\partial \pi}\right]_{C_{1}{ }^{0}, p=0}=\lim _{C_{1}^{\prime \prime} \rightarrow C_{1}}\left[\frac{\phi_{1}\left\{C_{1}, \pi\left(C_{1}, C_{1}^{\prime \prime}\right)\right\}}{\pi\left(C_{1}, C_{1}^{\prime \prime}\right)}\right]
$$

which is independent of the reference concentration $C_{1}^{0}$.

Analogous considerations hold for $\phi_{\mathrm{v}}$. The $\mathscr{L}_{\alpha \beta}$ coefficients can be obtained from such slopes taken from experimental flux curves built up over a set of concentration intervals.

When written with infinitesimal forces equations (29) and (30) become

$$
\begin{aligned}
& \partial \phi_{1}=\mathscr{L}_{\pi} \partial\left[\pi\left(1+\bar{C}_{1} V_{1}\right) / \bar{C}_{1}\right]+\mathscr{L}_{\pi \mathrm{p}} \partial(p-\pi) \\
& \partial \phi_{\mathrm{v}}=\mathscr{L}_{\mathrm{p} \alpha} \mathrm{\partial}\left[\pi\left(1+\bar{C}_{1} V_{1}\right) / C_{1}\right]+\mathscr{L}_{\mathrm{p}} \mathrm{\partial}(p-\pi)
\end{aligned}
$$

Here $\partial\left[\pi\left(1+\bar{C}_{1} V_{1}\right) / \bar{C}_{1}\right]$ implies the limit of $X_{1}$ as $C_{1} \rightarrow C_{1}^{0}$. 
From equation (26)

$$
\lim _{C_{1} \rightarrow C^{q}} \bar{C}_{1}=\frac{1}{V_{2}}\left(\frac{\partial \mu_{2}}{\partial \mu_{1}}\right)_{p, T}
$$

while from the Gibbs-Duhem equation

$$
C_{1} \partial \mu_{1}+C_{2} \partial \mu_{2}=C_{1} \partial \mu_{1}+\left(1-C_{1} V_{1}\right) \partial \mu_{2} / V_{2}=0
$$

Hence

$$
\lim _{C_{1} \rightarrow C_{1}^{0}} \bar{C}_{1}=C_{1} /\left(1-C_{1} V_{1}\right)
$$

and

$$
\lim _{C_{1} \rightarrow C_{1}^{0}}\left(1+\bar{C}_{1} V_{1}\right) / \bar{C}_{1}=1 / C_{1}
$$

Equations (35) and (36) may therefore be simplified to

$$
\begin{aligned}
& \partial \phi_{1}=\mathscr{L}_{\pi} \partial \pi / C_{1}+\mathscr{L}_{\pi \mathrm{p}} \partial(p-\pi) \\
& \partial \phi_{\mathrm{v}}=\mathscr{L}_{\mathrm{p} \pi} \partial \pi / C_{1}+\mathscr{L}_{\mathrm{p}} \mathrm{\partial}(p-\pi)
\end{aligned}
$$

\section{INTERDIFFUSION AND HYPERFILTRATION MEASUREMENTS}

For a series of interdiffusion experiments carried out under the restriction $p=0$ equations (41) and (42) give

$$
\begin{aligned}
& \left(\partial \phi_{1} / \partial \pi\right)_{p=0}=\mathscr{L}_{\pi} / C_{1}-\mathscr{L}_{\pi \mathrm{p}} \\
& \left(\partial \phi_{\mathrm{v}} / \partial \pi\right)_{p=0}=\mathscr{L}_{\mathrm{p} \pi} / C_{1}-\mathscr{L}_{\mathbf{p}}
\end{aligned}
$$

These equations give two relations between four $\mathscr{L}_{\alpha \beta}$ coefficients and the slopes of the experimental flux curves.

Two additional relations are needed to determine the coefficients. They are obtained from hyperfiltration experiments carried out at constant concentration $C_{1}$ on the high pressure side. Because the membrane has to be supported the composition of the solution on the low pressure side cannot be independently fixed. Once steady fluxes $\phi_{1}$ and $\phi_{\mathrm{v}}$ bave been set up the outgoing face of the membrane is always in contact with the product or effluent mixture which has concentration $C_{1}^{\mathrm{e}}$ given by

$$
C_{1}^{\mathrm{e}}=\phi_{1} / \phi_{\mathrm{v}}
$$

$C_{1}^{\mathrm{e}}$ and $\phi_{V}$ are measured so that $\phi_{1}$ can be calculated.

A series of measurements is made at constant $C_{1}^{0}$ by varying $p$. Several such series of measurements must be made with different ingoing concentrations $C_{1}^{0}$ so that $C_{1}^{\mathrm{e}}$ covers the whole composition range of interest. In each experiment $C_{1}^{0}$ and $C_{1}^{\mathrm{e}}$ are known; $\pi$ can therefore be calculated. Plots are drawn of $\phi_{1}, \phi_{\mathrm{v}}$ and $p$ versus $\pi$ at each constant $C_{1}^{0}$. These plots may be differentiated graphically at chosen values of $\pi$ and hence at particular values of $C_{1}$.

By comparing equations (29) and (30) with (31) and (32) for the same pair 
of solutions, i.e. at the same $C_{1}^{0}$ and $\pi$, it is seen that

$$
\begin{aligned}
& \left(\phi_{1} / \pi\right)=\left(\phi_{1} / \pi\right)_{p=0}+L_{i \mathrm{p}}(p / \pi) \\
& \left(\phi_{\mathrm{v}} / \pi\right)=\left(\phi_{\mathrm{v}} / \pi\right)_{p=0}+L_{\mathrm{p}}(p / \pi)
\end{aligned}
$$

Pursuing the same arguments as in the case of the interdiffusion experiments regarding extrapolation to vanishingly small $\pi$ at constant $C_{1}^{0}$ enables equations (46) and (47) to be converted to

$$
\begin{aligned}
& \mathscr{L}_{\pi \mathrm{p}}=\left[\left(\frac{\partial \phi_{1}}{\partial \pi}\right)_{c_{\mathrm{i}}^{0}}-\left(\frac{\partial \phi_{1}}{\partial \pi}\right)_{p=0}\right] \cdot\left(\frac{\partial \pi}{\partial p}\right)_{c_{1}^{o}} \\
& \mathscr{L}_{\mathbf{p}}=\left[\left(\frac{\partial \phi_{\mathrm{v}}}{\partial \pi}\right)_{c_{\mathrm{i}}^{0}}-\left(\frac{\partial \phi_{\mathrm{v}}}{\partial \pi}\right)_{p=0}\right] \cdot\left(\frac{\partial \pi}{\partial p}\right)_{c_{\mathrm{i}}^{o}}
\end{aligned}
$$

The differentials in these equations must all be evaluated at the same $C_{1}$. Equations (48) and (49) then permit $\mathscr{L}_{\pi \mathrm{p}}$ and $\mathscr{L}_{\mathrm{p}}$ to be evaluated for the membrane composition which is in equilibrium with solution of composition $C_{1}$. Values of $\mathscr{L}_{\pi}$ and $\mathscr{L}_{\mathrm{p} \pi}$ then follow immediately by using equations (43) and (44). This procedure may be repeated for many choices of $C_{1}$ so as to obtain the $\mathscr{L}_{\alpha \beta}$ coefficients as functions of concentration over the whole range of $C_{1}$ examined.

It is seen that making a combination of two kinds of transport experiments, interdiffusion between two different solutions under zero pressure difference and reverse osmosis or hyperfiltration measurements, over an appropriate range of concentrations and pressures permits the practical permeability coefficients $\mathscr{L}_{\mathrm{p}}$ and $\mathscr{L}_{\pi}$ together with the coupling coefficients $\mathscr{L}_{\mathrm{p} \pi}$ and $\mathscr{L}_{\pi \mathrm{p}}$ to be determined as functions of the solution concentration. If the coupling coefficients satisfy Onsager's relation

$$
\mathscr{L}_{\mathrm{p} \pi}=\mathscr{L}_{\pi \mathrm{p}}
$$

it is valuable evidence that the experiments have been carried out under conditions where the linear relations hold locally between fluxes and forces.

\section{EVALUATION OF MOLECULAR FRICTION COEFFICIENTS}

In order to convert the above coefficients to molecular friction coefficients in the polymer phase it is necessary to measure also the equilibrium sorption of components 1 and 2 by the polymer as a function of the composition of the bathing liquid mixture. From this information $C_{1}$ and $C_{2}$ in the solution may be related to $c_{1}$ and $c_{2}$ in the swollen membrane.

The following sequence of interconversions of the coefficients is also necessary

$$
\mathscr{L}_{\alpha \beta} \rightarrow \mathscr{L}_{i k} \rightarrow l_{i k} \rightarrow r_{i k} \rightarrow f_{i k}
$$

The procedure for doing this is explained in the following paragraphs.

The step $\mathscr{L}_{\alpha \beta} \rightarrow \mathscr{L}_{i k}$ follows from the formal transformation of particle fluxes $\phi_{1}$ and $\phi_{2}$ to practical fluxes $\phi_{1}$ and $\phi_{v}$ and the associated transformation of forces. The relation is

$$
\left(\mathscr{L}_{i k}\right)=\Gamma^{-1}\left(\mathscr{L}_{\alpha \beta}\right) \Gamma^{-1 T}
$$


where $\Gamma$ is the square matrix of equation (18). This gives the following expressions

$$
\begin{aligned}
& \mathscr{L}_{11}=\mathscr{L}_{\pi} \\
& \mathscr{L}_{12}=\left(\mathscr{L}_{\pi \mathrm{p}}-V_{1} \mathscr{L}_{\pi}\right) / V_{2} \\
& \mathscr{L}_{21}=\left(\mathscr{L}_{\mathrm{p} \pi}-V_{1} \mathscr{L}_{\pi}\right) / V_{2} \\
& \mathscr{L}_{22}=\left(\mathscr{L}_{\mathrm{p}}-V_{1} \mathscr{L}_{\mathrm{P} \pi}-V_{1} \mathscr{L}_{\pi \mathrm{p}}+V_{1}^{2} \mathscr{L}_{\pi}\right) / V_{2}^{2}
\end{aligned}
$$

These $\mathscr{L}_{i k}$ coefficients characterize the permeability properties that the actual membrane would have if it were studied under an infinitesimal potential difference at constant uniform composition. The coefficients are therefore inversely proportional to the membrane thickness $\delta$. The $l_{i k}$ coefficients measure the local permeability at unit gradient of potential. The relation between these sets of coefficients is

$$
\left(l_{i k}\right)=\delta\left(\mathscr{L}_{i k}\right)
$$

Note that there is a dimensional difference between $l_{i k}$ and $\mathscr{L}_{i k}$. The meaning of $\delta$ must be mentioned when the membrane is swollen by the solution. It is most appropriate to take for $\delta$ the thickness of the membrane when swollen to equilibrium with solution of the composition to which the $\mathscr{L}_{i k}$ are to refer. This choice must be borne in mind later when an interpretation of the concentration dependence of the $f_{i k}$ is attempted because, for example, the relations of the free volume theory of concentration dependent diffusion are written in terms either of the thermodynamic or of the intrinsic diffusion coefficient. The frame of reference corrections are by no means unimportant in highly swollen systems.

The connection between the $l_{i k}$ and the resistance coefficients $r_{i k}$ is given by equation (12). From equations (7), (8) and (9) the expressions for the friction coefficients follow. They are

$$
\begin{aligned}
& f_{12}=-c_{2} r_{12} \\
& f_{1 \mathbf{p}}=c_{1} r_{11}+c_{2} r_{12} \\
& f_{21}=-c_{1} r_{21} \\
& f_{2 \mathbf{p}}=c_{2} r_{22}+c_{1} r_{21}
\end{aligned}
$$

Here the concentrations $c_{1}$ and $c_{2}$ refer to the amounts of the components in the membrane when $\lrcorner$ wollen with a mixture of composition $C_{1}$ and $C_{2}$.

\section{PROCEDURE IN CASE OF PRESSURE DEPENDENCE}

It has been assumed throughout this discussion that a range of pressures exists over which the membrane properties are independent of the pressure while the fluxes are large enough to be measured. Little definite information is a vailable on this matter at present. In the reverse osmosis of water through cellulose acetate membranes the plots of flow versus pressure are linear up to quite high pressures ${ }^{30}$. Such membranes are only moderately swollen by water. Electrodialysis membranes are usually considerably more swollen and 
some non-linearity in the flux versus pressure curve for water occurs at pressures greater than $10 \mathrm{~atm}^{31}$. Very marked curvatures were obtained in the flux versus pressure curves for a number of organic liquids permeating a highly swollen lightly crosslinked rubber ${ }^{32}$.

The importance of pressure dependent flow properties appears to increase with increasing membrane swelling.

In view of these findings it will be prudent to determine flux versus pressure curves with each pure component 1 and 2 before embarking on hyperfiltration experiments recommended earlier. If the non-linearity is serious in the pressure range to be examined the experimental programme should be modified as follows.

Suppose it were desired to determine the frictional coefficients at some mixture composition $C_{1}$. A series of hyperfiltration experiments is carried out at some ingoing concentration $C_{1}^{0}$ and a series of effluent concentrations $C_{1}^{e}$ observed each corresponding to a different $p$. The concentration of interest $C_{1}$ must lie within the range of $C_{1}^{\mathrm{e}}$. $C_{1}$ would therefore be the actual effluent concentration at some pressure $p^{\prime}$ which can be estimated by interpolation. $\left(\partial \phi_{1} / \partial p\right)_{C_{1} \cdot} \cdot\left(\partial \phi_{v} / \partial p\right)_{C^{\circ}}$ and $(\partial \pi / \partial p)_{C_{1}^{0}}$ are measured at this pressure $p^{\prime}$.

A further set of measurements is made at some new $C_{1}^{0}$ closer to $C_{1}$. From these measurements a new pressure $p^{\prime \prime}$ at which $C_{1}$ would be the effluent concentration is found by interpolation. A second set of slopes is determined at this $p^{\prime \prime} . p^{\prime \prime}$ will be less than $p^{\prime}$ and repeating the procedure at a third $C_{1}^{0}$, still closer to $C_{1}$, will enable a third set of slopes to be obtained at a pressure $p^{\prime \prime \prime}$. Plotting each of the slopes against the relevant $p$ and extrapolating to $p=0$ permits the appropriate slopes needed for equations (48) and (49) to be evaluated. It may be anticipated that up to moderate pressures the permeability properties will be satisfactorily represented by a first order relation with $p$ and the extrapolation to $p=0$ would be uncomplicated. The calculation of the friction coefficients in the membrane at $p=0$ would then follow in the way described above.

\section{CONCLUSION}

The object of this paper has been to encourage all those who in the future may evaluate hyperfiltration as a means for separating mixtures of organic liquids to design their experimental programmes so as to be able to evaluate the molecular friction coefficients. Once a body of fundamental data of this kind has been amassed it will be possible to discover much about the flow mechanisms in this type of process. One may then know how the distribution of the components as dictated by the microstructure and morphology of the polymer as well as by the thermodynamic interactions of the mobile components with one another and with the polymer influence the degree of coupling between their fluxes.

It may also prove possible to make a significant assignment of the flow between contributions from random diffusional motions and from hydrodynamic flow processes taking place in liquid filled voids created by the swelling of a crosslinked network ${ }^{15}$. Only by achieving a mechanistic understanding of these processes will the ability to make predictions about the type of membrane most likely to effect a particular separation be developed. 


\section{PATRICK MEARES \\ REFERENCES}

1 Industrial Processing with Membranes. eds. R. E. Lacey and S. Loeb, Wiley-Interscience. New York. 1972.

2 A. S. Michaels and H. J. Bixler. Progr. Separation and Purification, ed. E. S. Perry. Interscience. New York 1968.

3 R. E. Kesting. Synthetic Polymeric Membranes, McGraw Hill, New York, 1971.

4 T. Teorell. Disc. Faraday Soc. 21, 9 (1956).

5 R. Schlögl, Stofftransport durch Membranen, Chap. 2. Steinkopf. Darmstadt (1964).

${ }^{6}$ P. Meares, J. Amer. Chem. Soc. 76. 3415 (1954).

7 H. B. Hopfenberg and V. Stannett. in The Physics of Glassy Polymers, ed. R. N. Haward. Chap. 9. Appl. Sci. Publ. : London (1973).

8 V. Stannett, in Diffusion in Polymers. eds. J. Crank and G. S. Park, Chap. 2. Academic Press : London (1968).

9 J. B. Alexopoulos, J. S. Barrie and D. Machin. Polymer. 10. 265 (1969).

${ }^{10}$ P. J. F. Kanitz and R. Y. M. Huang. J. Appl. Polymer S(i.. 14. 27.39 (197()).

11 S. A. Stern, S.-M. Fang and R. M. Jobbins, J. Macromol. Sci. (Physics), 5. 41 (1971).

12 H. K. Lonsdale in Desalination by Reverse Osmosis, ed. U. Merten, M.I.T. Press: Cambridge. Mass. (1966).

13 R. L. Riley. H. K. Lonsdale, C. R. Lyons and U. Merten. J. Appl. Polvmer Sci. 11. 2143 (1967).

14 G. Thau. R. Bloch and O. Kedem, Desalination, 1. 129 (1966).

15 P. Mieares, J. B. Craig and J. Webster, in Diffusion Processes, Proceedings of the Thomas Graham Memorial Symposium, Vol. 1, p. 609. Gordon and Breach, London (1970).

16 R. Y. M. Huang and V. J. C. Lin. J. Appl. Polymer. Sci. 12, 2615 (1968).

17 P. Meares. J. Polymer. Sci. 27, 391 (1958).

${ }^{18}$ H. Fujita, Fortschr. Hochpolym. Forsch. 3, 1 (1961).

19 D. Machin and C. E. Rogers, Makromol. Chem. 155, 269 (1972).

${ }^{20}$ R. M. Barrer, R. Mallinder and P. S. C. Wong, Polymer, 8, 321 (1967).

21 M. Fels and R. Y. M. Huang. J. Macromol. Sci. (Physics). 5. 89 (1971).

22 A. S. Michaels, W. Vieth, A. S. Hoffman and H. A. Alcalay, J. Appl. Polymer Sci. 13, 577 (1969)

${ }_{23}$ R. D. Siegel and R. W. Coughlin, Nature, 226, 938 (1970).

${ }^{24}$ S. Sourirajan. Reverse Osmosis, Chap. 7. Logos, London (1970).

25 L. Onsager, Ann. N.Y. Acad. Sci. 46, 241 (1945).

26 A. Katchalsky and O. Kedem, Biophys. J. 2, 53 (1962).

27 R. L. Riley, U. Merten and J. O. Gardner, Desalination, 1, 30 (1966).

28 H. Kramer and P. Meares, Biophys. J. 9, 1006 (1969).

${ }^{29}$ A. Zelman, J. C. T. Kwak, J. Leibovitz and K. S. Spiegler. Biol. Aspects of Electrochem. Experentia Suppl. 18, p. 679. Birkhauser. Basel (1971).

${ }^{30}$ S. Sourirajan. Reverse Osmosis, Chap. 3. Logos. London (1970).

31 W. Pusch, Proc. 3rd Int. Symp. Freshwater from the Sea. 2. $535(1970)$.

32 D. R. Paul and O. M. Ebra-Lima, J. Appl. Polymer Sci. 14, 2201 (1970). 